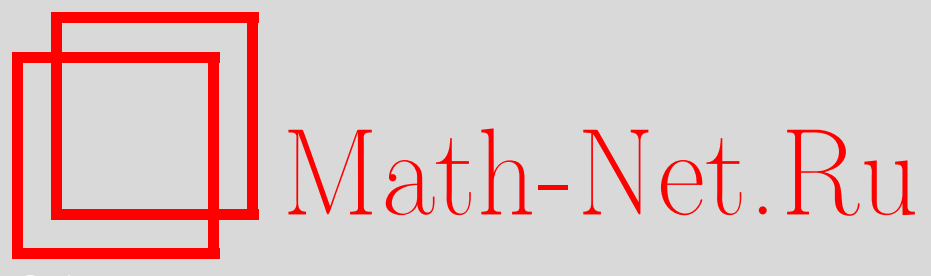

$\Phi$. Ветро, О накрытиях со специальными слоями и группой монодромии $S_{d}$, Изв. РАН. Сер. матем., 2012, том 76, выпуск 6, 39-44

DOI: https://doi.org/10.4213/im7862

Использование Общероссийского математического портала Math-Net.Ru подразумевает, что вы прочитали и согласны с пользовательским соглашением http://www . mathnet.ru/rus/agreement

Параметры загрузки:

IP : 3.89 .197 .203

26 апреля 2023 г., 16:27:59

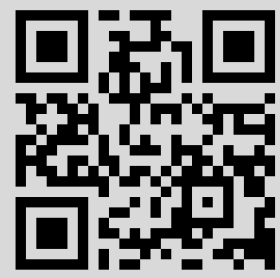




\title{
Ф. Ветро
}

\section{О накрытиях со специальными слоями и группой монодромии $S_{d}$}

\begin{abstract}
Рассматриваются разветвленные накрытия степени $d$ над $Y$ с группой монодромии $S_{d}, k$ простыми точками ветвления, $n-k$ специальными точками и фиксированными данными ветвления в специальных точках, где $Y$ - гладкая связная комплексная проективная кривая рода $g \geqslant 1$, а $n$ и $k-$ целые числа такие, что $n>k>0$. Показано, что соответствующие пространства Гурвица неприводимы при $k>3 d-3$.
\end{abstract}

Библиография: 15 наименований.

Ключевые слова: пространства Гурвица, специальные слои, разветвленные накрытия, монодромия, действия кос.

\section{Введение}

Вычисление неприводимых компонент пространств Гурвица - одна из классических задач алгебраической геометрии. Первый результат в этой области принадлежит самому Гурвицу и состоит в том, что пространство Гурвица общих накрытий над $\mathbb{P}^{1}$ степени $d$ с $n$ точками ветвления неприводимо (см. [1]). За последние 30 лет было получено много обобщений этого результата (см., например, [2]-[9]). В работе Вик. С. Куликова [5] рассматриваются накрытия над $\mathbb{P}^{1}$ степени $d$ с группой монодромии $S_{d}, k$ простейшими точками ветвления (т. е. с ветвлением 2-го порядка) и $n-k$ специальными точками, фиксируются данные ветвления в специальных точках и доказывается неприводимость соответствующих пространств Гурвица при $k>3 d-3$ [5, теорема 3.3]. В настоящей статье этот результат распространяется на накрытия гладких связных комплексных проективных кривых рода не менее 1. Мы доказываем, что соответствующие пространства Гурвица неприводимы при $k>3 d-3$, и тем самым обобщаем результат Вик. С. Куликова в случае кривых рода не менее 1.

Разветвленные накрытия $f_{1}: X_{1} \rightarrow Y$ и $f_{2}: X_{2} \rightarrow Y$ степени $d$ над $Y$ называются эквивалентными, если существует биголоморфное отображение $\pi: X_{1} \rightarrow X_{2}$ такое, что $f_{2} \circ \pi=f_{1}$. Класс эквивалентности накрытия $f_{1}$ обозначается через $\left[f_{1}\right]$. Будем также обозначать перестановку $h^{-1} t h$ через $t^{h}$, а подгруппу в $S_{d}$, порожденную перестановками $t_{1}, \ldots, t_{l}$, через $\left\langle t_{1}, \ldots, t_{l}\right\rangle$.

\section{§ 1. Пространства Гурвица $H_{d, k, q_{1} C_{1}, \ldots, q_{r} C_{r}}^{S_{d}}(Y)$}

Пусть $X, Y$ - гладкие связные комплексные проективные кривые, причем род $Y$ равен $g$, и пусть $d, n, k$ - целые числа, $d \geqslant 3, n>k>0$. Мы рассматриваем разветвленные накрытия $f: X \rightarrow Y$ степени $d$ над $Y$ с группой монодромии $S_{d}$, имеющие $k$ простых точек ветвления и $n-k$ специальных точек. 
Пусть $C_{1}, \ldots, C_{r}$ - классы сопряженности в $S_{d}$, не содержащие перестановок $(1,2)$ и id, a $q_{1}, \ldots, q_{r}$ - положительные целые числа такие, что

$$
q_{1}+\cdots+q_{r}=n-k .
$$

Обозначим через $H_{d, k, q_{1} C_{1}, \ldots, q_{r} C_{r}}^{S_{d}}(Y)$ пространство Гурвица классов эквивалентности описанных выше накрытий, локальная монодромия которых при каждом $i=1, \ldots, r$ в ровно $q_{i}$ из $n-k$ специальных точках принадлежит $C_{i}$.

Фиксируем точку $b_{0}$ кривой $Y$ и конечное подмножество $D$ в $Y$ такие, что $b_{0} \in Y-D$. По теореме Римана о существовании накрытий (см. [10, предложение 1.2]) имеется взаимно однозначное соответствие между:

(i) множеством классов эквивалентности разветвленных накрытий степени $d$ над $Y$ с множеством точек ветвления $D$;

(ii) множеством классов эквивалентности гомоморфизмов $m: \pi_{1}\left(Y-D, b_{0}\right) \rightarrow$ $S_{d}$ с образом, равным транзитивной подгруппе группы $S_{d}$, причем гомоморфизмы $m$ и $m^{\prime}$ эквивалентны, если существует перестановка $h \in S_{d}$ такая, что $m^{\prime}([\gamma])=h^{-1} m([\gamma]) h$ для всех $[\gamma] \in \pi_{1}\left(Y-D, b_{0}\right)$.

Далее через $D$ и $m$ обозначаются соответственно множество точек ветвления и гомоморфизм монодромии накрытия $f$.

ОПРЕДЕЛЕНИЕ 1. Упорядоченный набор $\left(t_{1}, \ldots, t_{n} ; \lambda_{1}, \mu_{1}, \ldots, \lambda_{g}, \mu_{g}\right):=$ $(\underline{t}, \underline{\lambda}, \underline{\mu})$ перестановок из $S_{d}$ называется системой Гурвии $a$, если $t_{i} \neq \mathrm{id}$ ни для какого $i \in\{1, \ldots, n\}$ и $t_{1} \cdots t_{n}=\left[\lambda_{1}, \mu_{1}\right] \cdots\left[\lambda_{g}, \mu_{g}\right]$. Подгруппа в $S_{d}$, порожденная элементами $t_{i}, \lambda_{s}, \mu_{s}, i=1, \ldots, n, s=1, \ldots, g$, называется группой монодромии данной системы Гурвица. Две системы Гурвица $(\underline{t}, \underline{\lambda}, \underline{\mu})$ и $\left(\underline{t}^{\prime}, \underline{\lambda}^{\prime}, \underline{\mu}^{\prime}\right)$ считаются эквивалентными, если существует перестановка $h \in \bar{S}_{d}$ такая, что $t_{i}^{\prime}=h^{-1} t_{i} h, \lambda_{s}^{\prime}=h^{-1} \lambda_{s} h$ и $\mu_{s}^{\prime}=h^{-1} \mu_{s} h$ для всех $i=1, \ldots, n$ и $s=1, \ldots, g$.

ЗАмЕчАниЕ 1. Упорядоченный набор $\left(t_{1}, \ldots, t_{n}\right)$ перестановок из $S_{d}$ (при $t_{i} \neq \mathrm{id}$ ни для какого $i$ ) является системой Гурвица, если $t_{1} \cdots t_{n}=\mathrm{id}$.

Пусть $\left(\gamma_{1}, \ldots, \gamma_{n} ; \alpha_{1}, \beta_{1}, \ldots, \alpha_{g}, \beta_{g}\right)$ - стандартная система образующих группы $\pi_{1}\left(Y-D, b_{0}\right)$. Образы элементов $\gamma_{1}, \ldots, \gamma_{n}, \alpha_{1}, \beta_{1}, \ldots, \alpha_{g}, \beta_{g}$ при гомоморфизме $m$ задают класс эквивалентности систем Гурвица $\left[t_{1}, \ldots, t_{n} ; \lambda_{1}, \mu_{1}, \ldots\right.$ $\left.\ldots, \lambda_{g}, \mu_{g}\right]$ с группой монодромии $S_{d}$ таких, что $k$ перестановок $t_{i}$ - транспозиции и ровно $q_{i}$ элементов $t_{i}$ принадлежат $C_{i}$ при $i=1, \ldots, r$. Обозначим множество классов эквивалентности всех таких систем Гурвица через $A_{k, q_{1} C_{1}, \ldots, q_{r} C_{r}, g}^{0}$ Заметим, что при фиксированных замкнутых кривых $\left(\gamma_{1}, \ldots, \beta_{g}\right)$ указанного выше вида теорема Римана о существовании позволяет нам отождествить $A_{k, q_{1} C_{1}, \ldots, q_{r} C_{r}, g}^{0}$ с множеством классов эквивалентности $[f] \in H_{d, k, q_{1} C_{1}, \ldots, q_{r} C_{r}}^{S_{d}}(Y)$ таких, что $f$ имеет множество ветвления $D$.

\section{§ 2. Действия кос}

Далее через $Y^{(n)}$ обозначается $n$-я симметрическая степень кривой $Y$, а через $\Delta$ - подмножество коразмерности 1 в $Y^{(n)}$, состоящее из всех непростых дивизоров. В этом параграфе мы напоминаем, как образующие группы кос $\pi_{1}\left(Y^{(n)}-\Delta, D\right)$ действуют на системах Гурвица. Это действие изучали А. Гурвиц [1], Т. Граббер, Дж. Харрис, Дж. Старр [11] и В. Канев [3].

Образующими группы кос $\pi_{1}\left(Y^{(n)}-\Delta, D\right)$ являются элементарные косы $\sigma_{i}$, $i=1, \ldots, n-1$, а также косы $\rho_{j s}, \tau_{j s}$, где $1 \leqslant j \leqslant n$ и $1 \leqslant s \leqslant g$ (см. [12]-[14]). 
$\mathrm{C}$ каждой образующей $\sigma_{i}$ связаны два преобразования $\sigma_{i}^{\prime}$ и $\sigma_{i}^{\prime \prime}=\left(\sigma_{i}^{\prime}\right)^{-1}$, называемые элементарными преобразованиями и действующие следующим образом. Все элементы $\lambda_{s}$, все $\mu_{s}$ и $t_{h}$ при $h \neq i, i+1$ остаются на месте, а пара $\left(t_{i}, t_{i+1}\right)$ переходит при $\sigma_{i}^{\prime}, \sigma_{i}^{\prime \prime}$ в пары $\left(t_{i} t_{i+1} t_{i}^{-1}, t_{i}\right),\left(t_{i+1}, t_{i+1}^{-1} t_{i} t_{i+1}\right)$ соответственно (см. [1]). Обозначим через

$$
\rho_{j s}^{\prime}, \rho_{j s}^{\prime \prime}=\left(\rho_{j s}^{\prime}\right)^{-1}, \quad \tau_{j s}^{\prime}, \tau_{j s}^{\prime \prime}=\left(\tau_{j s}^{\prime}\right)^{-1}
$$

пары преобразований, отвечающих косам $\rho_{j s}$ и $\tau_{j s}$ соответственно. Нам понадобится следующий результат.

ПРЕДЛОЖКеНИЕ 1 [3, теорема 1.8]. Пусть $\left(t_{1}, \ldots, t_{n} ; \lambda_{1}, \mu_{1}, \ldots, \lambda_{g}, \mu_{g}\right)-c u$ стема Гурвииа. Положим $u_{0}=\mathrm{id}, u_{s}=\left[\lambda_{1}, \mu_{1}\right] \cdots\left[\lambda_{s}, \mu_{s}\right]$ nрu $s=1, \ldots, g$. Тогда действия кос

$$
\left(t_{1}, \ldots, t_{n} ; \lambda_{1}, \mu_{1}, \ldots, \lambda_{g}, \mu_{g}\right) \rightarrow\left(t_{1}^{\prime}, \ldots, t_{n}^{\prime} ; \lambda_{1}^{\prime}, \mu_{1}^{\prime}, \ldots, \lambda_{g}^{\prime}, \mu_{g}^{\prime}\right)
$$

задаются следующим образом:

1) для $\rho_{i s}^{\prime}$ при $1 \leqslant i \leqslant n, 1 \leqslant s \leqslant g$ имеем $t_{j}^{\prime}=t_{j}$ для всех $j \neq i, \lambda_{l}^{\prime}=\lambda_{l}$ для всех $l, \mu_{l}^{\prime}=\mu_{l}$ для всех $l \neq s$,

$$
\left(t_{i}, \mu_{s}\right) \rightarrow\left(t_{i}^{\prime}, \mu_{s}^{\prime}\right)=\left(a_{1}^{-1} t_{i} a_{1}, b_{1}^{-1} t_{i}^{-1} b_{1} \mu_{s}\right),
$$

где

$$
a_{1}=\left(t_{1} \cdots t_{i-1}\right)^{-1} u_{s-1} \lambda_{s}\left(u_{s}^{-1} u_{g}\right)\left(t_{i+1} \cdots t_{n}\right)^{-1}, \quad b_{1}=\left(t_{1} \cdots t_{i-1}\right)^{-1} u_{s-1} \lambda_{s} ;
$$

2) для $\tau_{i s}^{\prime}$ при $1 \leqslant i \leqslant n, 1 \leqslant s \leqslant g$ имеем $t_{j}^{\prime}=t_{j}$ для всех $j \neq i, \lambda_{l}^{\prime}=\lambda_{l}$ для всех $l \neq s, \mu_{l}^{\prime}=\mu_{l}$ для всех $l$,

$$
\left(t_{i}, \lambda_{s}\right) \rightarrow\left(t_{i}^{\prime}, \lambda_{s}^{\prime}\right)=\left(c_{1}^{-1} t_{i} c_{1}, d_{1}^{-1} t_{i} d_{1} \lambda_{s}\right)
$$

əдe

$$
c_{1}=t_{i+1} \cdots t_{n}\left(u_{s}^{-1} u_{g}\right)^{-1} \mu_{s}\left(u_{s-1}\right)^{-1} t_{1} \cdots t_{i-1}, \quad d_{1}=t_{i+1} \cdots t_{n}\left(u_{s}^{-1} u_{g}\right)^{-1} \mu_{s} \text {; }
$$

3) для $\rho_{i s}^{\prime \prime}$ при $1 \leqslant i \leqslant n, 1 \leqslant s \leqslant g$ имеем $t_{j}^{\prime}=t_{j}$ для всех $j \neq i, \lambda_{l}^{\prime}=\lambda_{l}$ для всех $l, \mu_{l}^{\prime}=\mu_{l}$ для всех $l \neq s$,

$$
\left(t_{i}, \mu_{s}\right) \rightarrow\left(t_{i}^{\prime}, \mu_{s}^{\prime}\right)=\left(a_{2}^{-1} t_{i} a_{2}, b_{2}^{-1} t_{i} b_{2} \mu_{s}\right)
$$

где

$$
a_{2}=t_{i+1} \cdots t_{n}\left(u_{s}^{-1} u_{g}\right)^{-1} \lambda_{s}^{-1}\left(u_{s-1}\right)^{-1} t_{1} \cdots t_{i-1}, \quad b_{2}=t_{i+1} \cdots t_{n}\left(u_{s}^{-1} u_{g}\right)^{-1} ;
$$

4) для $\tau_{i s}^{\prime \prime}$ nрu $1 \leqslant i \leqslant n, 1 \leqslant s \leqslant g$ имеем $t_{j}^{\prime}=t_{j}$ для всех $j \neq i, \lambda_{l}^{\prime}=\lambda_{l}$ для всех $l \neq s, \mu_{l}^{\prime}=\mu_{l}$ для всех $l$,

$$
\left(t_{i}, \lambda_{s}\right) \rightarrow\left(t_{i}^{\prime}, \lambda_{s}^{\prime}\right)=\left(c_{2}^{-1} t_{i} c_{2}, d_{2}^{-1} t_{i}^{-1} d_{2} \lambda_{s}\right)
$$

где

$$
c_{2}=\left(t_{1} \cdots t_{i-1}\right)^{-1} u_{s-1} \mu_{s}^{-1}\left(u_{s}^{-1} u_{g}\right)\left(t_{i+1} \cdots t_{n}\right)^{-1}, \quad d_{2}=\left(t_{1} \cdots t_{i-1}\right)^{-1} u_{s-1} .
$$

Заметим, что преобразования $\rho_{j s}^{\prime}, \rho_{j s}^{\prime \prime}, \tau_{j s}^{\prime}$ и $\tau_{j s}^{\prime \prime}$ переводят $t_{j}$ в элементы из того же класса сопряженности. Кроме того, если $\lambda_{1}=\cdots=\lambda_{s}=\mu_{1}=\cdots$ $\cdots=\mu_{s-1}=\mathrm{id}$, то $\rho_{1 s}^{\prime}$ переводит $\mu_{s}$ в $t_{1}^{-1} \mu_{s}$.

Аналогично, если $\lambda_{1}=\cdots=\lambda_{s-1}=\mu_{1}=\cdots=\mu_{s-1}=\mathrm{id}$, то $\tau_{1 s}^{\prime \prime}$ переводит $\lambda_{s}$ в $t_{1}^{-1} \lambda_{s}$. 
ЗАмЕчаниЕ 2. Действия образующих $\rho_{j s}$ и $\tau_{j s}$ подробно изучались в работе [11], где действия кос, аналогичные используемым нами, применялись, в частности, для доказательства неприводимости пространств Гурвица простых накрытий с полной группой монодромии над кривыми положительного рода. С помощью этих результатов в [15] было доказано существование сечений однопараметрического семейства комплексных рационально связных алгебраических многообразий.

ОПреДЕЛЕНиЕ 2. Две системы Гурвица называются брэйд-эквивалентнъми, если одна из них получается из другой конечным числом преобразований $\sigma_{i}^{\prime}, \rho_{j s}^{\prime}, \tau_{j s}^{\prime}, \sigma_{i}^{\prime \prime}, \rho_{j s}^{\prime \prime}, \tau_{j s}^{\prime \prime}$, где $1 \leqslant i \leqslant n-1,1 \leqslant j \leqslant n$ и $1 \leqslant s \leqslant g$. Два упорядоченных набора перестановок $\left(t_{1}, \ldots, t_{l}\right)$ и $\left(t_{1}^{\prime}, \ldots, t_{l}^{\prime}\right)$ называются брэйд-эквивалентными, если $\left(t_{1}^{\prime}, \ldots, t_{l}^{\prime}\right)$ получается из $\left(t_{1}, \ldots, t_{l}\right)$ конечным числом преобразований вида $\sigma_{i}^{\prime}, \sigma_{i}^{\prime \prime}$. Будем обозначать брэйд-эквивалентность через $\sim$.

\section{§ 3. Неприводимость $H_{d, k, q_{1} C_{1}, \ldots, q_{r} C_{r}}^{S_{d}}(Y)$}

Далее $Y$ - гладкая связная комплексная проективная кривая рода $g \geqslant 1$. Пусть $\underline{e}=\left(e_{1}, \ldots, e_{s}\right)$ - разбиение числа $d$, задающее цикловый тип перестановок из $C_{1}$. Через $|\underline{e}|$ обозначим число $\sum_{i=1}^{s}\left(e_{i}-1\right)$.

Нам потребуются следующие результаты.

Лемма 1 [8, предложение 3]. Пусть $\left(t_{1}, t_{2}, \ldots, t_{l}\right)$ - такой набор перестановок из $S_{d}$, что $t_{1}$ имеет цикловый тип $\underline{e}$, a $t_{2}, \ldots, t_{l}$ - транспозиции. Если $l-1+|\underline{e}| \geqslant 2 d$, то набор $\left(t_{1}, t_{2}, \ldots, t_{l}\right)$ брэйд-эквивалентен набору $\left(t_{1}^{\prime}, t_{2}^{\prime}, \ldots, t_{l-2}^{\prime}, t_{l-1}^{\prime}, t_{l}^{\prime}\right)$ такому, что $t_{1}^{\prime}$ имеет иикловый тип $\underline{e}, t_{2}^{\prime}, \ldots, t_{l}^{\prime}-$ транспозичии, $t_{l-1}^{\prime}=t_{l}^{\prime} u\left\langle t_{1}^{\prime}, t_{2}^{\prime}, \ldots, t_{l-2}^{\prime}\right\rangle=\left\langle t_{1}^{\prime}, \ldots, t_{l-2}^{\prime}, t_{l-1}^{\prime}, t_{l}^{\prime}\right\rangle$.

Лемма 2 [3, лемма 2.1]. Пусть $\left(t_{1}, \ldots, t_{n} ; \lambda_{1}, \mu_{1}, \ldots, \lambda_{g}, \mu_{g}\right)$ - система Гурвица перестановок из $S_{d}$. Допустим, что $t_{i} t_{i+1}=\mathrm{id}, u$ обозначим через $H$ подгруппу $S_{d}$, порожденную элементами

$$
\left\{t_{1}, \ldots, t_{i-1}, t_{i+2}, \ldots, t_{n}, \lambda_{1}, \mu_{1}, \ldots, \lambda_{g}, \mu_{g}\right\} .
$$

Тогда для любого $h \in H$ исходная система Гурвица брэйд-эквивалентна системе $\left(t_{1}, \ldots, t_{i-1}, t_{i}^{h}, t_{i+1}^{h}, t_{i+2}, \ldots, t_{n} ; \lambda_{1}, \mu_{1}, \ldots, \lambda_{g}, \mu_{g}\right)$.

Теорема 1 [5, теорема 3.3]. Пространство Гурвица $H_{d, k, q_{1} C_{1}, \ldots, q_{r} C_{r}}^{S_{d}}\left(\mathbb{P}^{1}\right)$ неприводимо при $k>3 d-3$.

С помощью лемм 1, 2 мы обобщим результат теоремы 1 в случае кривых рода не менее 1.

Теорема 2. Пространство Гурвица $H_{d, k, q_{1} C_{1}, \ldots, q_{r} C_{r}}^{S_{S_{2}}}(Y)$ неприводимо при $k>3 d-3$.

ДоказАТЕЛьство. Пусть отображение $\delta: H_{d, k, q_{1} C_{1}, \ldots, q_{r} C_{r}}^{S_{d}}(Y) \rightarrow Y^{(n)}-\Delta$ сопоставляет каждому классу эквивалентности накрытий $[f]$ множество точек ветвления накрытия $f$. Как отмечено в [10], топология пространства $H_{d, k, q_{1} C_{1}, \ldots, q_{r} C_{r}}^{S_{d}}(Y)$ такова, что $\delta$ - топологическое накрытие. Поэтому группа кос $\pi_{1}\left(Y^{(n)}-\Delta, D\right)$ действует на $A_{k, q_{1} C_{1}, \ldots, q_{r} C_{r}, g}^{0}$ (см. $\left.\S 1\right)$. Орбиты этого 
действия находятся во взаимно однозначном соответствии со связными компонентами пространства $H_{d, k, q_{1} C_{1}, \ldots, q_{r} C_{r}}^{S_{d}}(Y)$. Поскольку это пространство гладко, для доказательства его неприводимости достаточно установить, что оно связно, т. е. что группа $\pi_{1}\left(Y^{(n)}-\Delta, D\right)$ действует на $A_{k, q_{1} C_{1}, \ldots, q_{r} C_{r}, g}^{0}$ транзитивно. Для доказательства этой транзитивности, в свою очередь, достаточно установить, что каждый класс эквивалентности систем Гурвица из $A_{k, q_{1} C_{1}, \ldots, q_{r} C_{r}, g}^{0}$ брэйд-эквивалентен классу вида $[\underline{\tilde{t}} ; \mathrm{id}, \ldots, \mathrm{id}]$. Действительно, поскольку класс эквивалентности $[\underline{\tilde{t}}]$ принадлежит $A_{k, q_{1} C_{1}, \ldots, q_{r} C_{r}, 0}^{0}$, требуемое утверждение будет вытекать из теоремы 1.

Шаг 1. Пусть $[\underline{t} ; \underline{\lambda}, \underline{\mu}] \in A_{k, q_{1} C_{1}, \ldots, q_{r} C_{r}, g}^{0}$ и $\eta$ - любая транспозиция из $S_{d}$. Покажем, что класс $[\underline{t} ; \underline{\bar{\lambda}}, \underline{\mu}]$ брэйд-эквивалентен классу вида $[\ldots, \eta, \eta, \ldots ; \underline{\lambda}, \underline{\mu}]$.

Элементарными преобразованиями вида $\sigma_{i}^{\prime \prime}$ можно передвинуть одну из перестановок класса сопряженности $C_{1}$ на первое место, а транспозиции - на места с номерами $2, \ldots, k+1$. Таким образом, набор $\underline{t}$ брэйд-эквивалентен набору вида $\left(t_{1}^{\prime}, t_{2}^{\prime}, \ldots, t_{k+1}^{\prime}, \ldots, t_{n}^{\prime}\right)$, где $t_{1}^{\prime}$ принадлежит $C_{1}$, а $t_{2}^{\prime}, \ldots, t_{k+1}^{\prime}-$ транспозиции.

Из предположения $k>3 d-3$ вытекает, что $k+|\underline{e}|>3 d-3 \geqslant 2 d$. Поэтому набор перестановок $\left(t_{1}^{\prime}, t_{2}^{\prime}, \ldots, t_{k+1}^{\prime}\right)$ удовлетворяет всем условиям леммы 1 и, следовательно, брэйд-эквивалентен набору вида $\left(t_{1}^{\prime \prime}, t_{2}^{\prime \prime}, \ldots, t_{k+1}^{\prime \prime}\right)$, где $t_{1}^{\prime \prime}$ имеет цикловый тип $\underline{e}, t_{2}^{\prime \prime}, \ldots, t_{k+1}^{\prime \prime}-$ транспозиции, $t_{k}^{\prime \prime}=t_{k+1}^{\prime \prime}$ и

$$
\left\langle t_{1}^{\prime \prime}, t_{2}^{\prime \prime}, \ldots, t_{k-1}^{\prime \prime}\right\rangle=\left\langle t_{1}^{\prime \prime}, \ldots, t_{k-1}^{\prime \prime}, t_{k}^{\prime \prime}, t_{k+1}^{\prime \prime}\right\rangle .
$$

Поскольку группа монодромии системы Гурвица $\left(\underline{t}^{\prime \prime} ; \underline{\lambda}, \underline{\mu}\right)$ равна $S_{d}$, по лемме 2 класс $\left[\underline{t}^{\prime \prime} ; \underline{\lambda}, \underline{\mu}\right]$ брэйд-эквивалентен классу вида $[\ldots, \bar{\eta}, \eta, \ldots ; \underline{\lambda}, \underline{\mu}]$, где $\eta-$ произвольная транспозиция из $S_{d}$, что и требовалось доказать.

Шаг 2. Покажем, что любой класс $\left[\underline{t} ; \lambda_{1}, \mu_{1}, \ldots, \lambda_{g}, \mu_{g}\right] \in A_{k, q_{1} C_{1}, \ldots, q_{r} C_{r}, g}^{0}$ брэйд-эквивалентен классу вида $[\tilde{t} ; \mathrm{id}, \ldots, \mathrm{id}]$.

Воспользуемся индукцией по $\sum_{h=1}^{g}\left(\left|\lambda_{h}\right|+\left|\mu_{h}\right|\right)$. Если $\sum_{h=1}^{g}\left(\left|\lambda_{h}\right|+\left|\mu_{h}\right|\right)=0$, то $\lambda_{h}=$ id и $\mu_{h}=$ id для всех $h=1, \ldots, g$. Поэтому будем считать, что $\sum_{h=1}^{g}\left(\left|\lambda_{h}\right|+\left|\mu_{h}\right|\right)>0$. Тогда по крайней мере одна из перестановок $\lambda_{h}$ или $\mu_{h}$ отлична от id.

Если $\lambda_{1} \neq \mathrm{id}$, то пусть $\eta$ - такая транспозиция, что $\left|\eta \lambda_{1}\right|=\left|\lambda_{1}\right|-1$. Согласно шагу 1 , действуя лишь элементарными преобразованиями и не пользуясь сопряжением, можно заменить класс $\left[\underline{t} ; \lambda_{1}, \mu_{1}, \ldots, \lambda_{g}, \mu_{g}\right]$ на класс вида $[\ldots, \eta, \eta, \ldots ; \underline{\lambda}, \underline{\mu}]$. После этого мы переносим одну из транспозиций $\eta$ на первое место и применяем $\tau_{11}^{\prime \prime}$. Тогда $\lambda_{1}$ преобразуется в $\lambda_{1}^{\prime}=\eta \lambda_{1}$, причем $\left|\lambda_{1}^{\prime}\right|<\left|\lambda_{1}\right|$. Теперь требуемое утверждение вытекает из предположения индукции.

Если $\lambda_{1}=$ id и $\mu_{1} \neq \mathrm{id}$, то выберем транспозицию $\eta$ такую, что $\left|\eta \mu_{1}\right|<\left|\mu_{1}\right|$. Согласно шагу 1 , действуя лишь элементарными преобразованиями, заменим $\left[\underline{t} ; \lambda_{1}, \mu_{1}, \ldots, \lambda_{g}, \mu_{g}\right]$ на класс вида $[\ldots, \eta, \eta, \ldots ; \underline{\lambda}, \underline{\mu}]$. Опять перенесем одну из транспозиций $\eta$ на первое место, но подействуем теперь преобразованием $\rho_{11}^{\prime}$. Тогда $\mu_{1}$ преобразуется в $\mu_{1}^{\prime}=\eta \mu_{1}$ и требуемое утверждение вытекает из предположения индукции.

Если $\lambda_{s} \neq \mathrm{id}$ и $\lambda_{1}=\cdots=\lambda_{s-1}=\mu_{1}=\cdots=\mu_{s-1}=\mathrm{id}$, то действуем аналогично, но применяем на этот раз преобразование $\tau_{1 s}^{\prime \prime}$, чтобы перевести $\lambda_{s}$ в $\eta \lambda_{s},\left|\eta \lambda_{s}\right|<\left|\lambda_{s}\right|$. Точно так же, если $\mu_{s} \neq$ id и $\lambda_{1}=\cdots=\lambda_{s}=\mu_{1}=\cdots$ $\cdots=\mu_{s-1}=\mathrm{id}$, применяем $\rho_{1 s}^{\prime}$, чтобы перевести $\mu_{s}$ в $\eta \mu_{s},\left|\eta \mu_{s}\right|<\left|\mu_{s}\right|$. В этих двух случаях требуемое утверждение последует из предположения индукции. 
Шаг 3. На шаге 2 мы показали, что любой класс $\left[\underline{t} ; \lambda_{1}, \mu_{1}, \ldots, \lambda_{g}, \mu_{g}\right] \in$ $A_{k, q_{1} C_{1}, \ldots, q_{r} C_{r}, g}^{0}$ брэйд-эквивалентен классу вида $[\underline{\tilde{t}} ; \mathrm{id}, \ldots, \mathrm{id}]$. Заметим теперь, что класс эквивалентности $[\underline{\tilde{t}}]$ принадлежит $A_{k, q_{1} C_{1}, \ldots, q_{r} C_{r}, 0}^{0}$. По теореме Римана о существовании этот класс отвечает некоторому классу эквивалентности накрытий из $H_{d, k, q_{1} C_{1}, \ldots, q_{r} C_{r}}^{S_{d}}\left(\mathbb{P}^{1}\right)$. Поскольку $k>3 d-3$, пространство Гурвица $H_{d, k, q_{1} C_{1}, \ldots, q_{r} C_{r}}^{S_{d}}\left(\mathbb{P}^{1}\right)$ неприводимо по теореме 1.

Теорема доказана.

\section{Список литературы}

1. A. Hurwitz, "Ueber Riemann'sche Flächen mit gegebenen Verzweigungspunkten", Math. Ann., 39:1 (1891), 1-60.

2. I. Berstein, A.L. Edmonds, "On the classification of generic branched coverings of surfaces", Illinois J. Math., 28:1 (1984), 64-82.

3. V. Kanev, Irreducibility of Hurwitz spaces, Preprint № 241, Dipartimento di Matematica ed Applicazioni, Università di Palermo, 2004; arXiv: math/0509154.

4. P. Kluitmann, "Hurwitz action and finite quotients of braid groups", Braids (Santa Cruz, CA, 1986), Contemp. Math., 78, Amer. Math. Soc., Providence, RI, 1988, 299-325.

5. Вик. С. Куликов, "Полугруппы разложений на множители и неприводимые компоненты пространства Гурвица", Изв. РАН. Сер. матем., 75:4 (2011), 49-90; англ. пер.: V.S. Kulikov, "Factorization semigroups and irreducible components of the Hurwitz space", Izv. Math., 75:4 (2011), 711-748.

6. S. Mochizuki, "The geometry of the compactification of the Hurwitz scheme", Publ. Res. Inst. Math. Sci., 31:3 (1995), 355-441.

7. С. М. Натанзон, "Топология двумерных накрытий и мероморфные функции на вещественных и комплексных алгебраических кривых", Труды семинара по векторному и тензорному анализу, 1988, № 23, 79-103; англ. пер.: S. M. Natanzon, "Topology of 2-dimensional coverings and meromorphic functions on real and complex algebraic curves", Selecta Math. Soviet., 12:3 (1993), 251-291.

8. F. Vetro, "Irreducibility of Hurwitz spaces of coverings with one special fiber", Indag. Math. (N.S.), 17:1 (2006), 115-127.

9. B. Wajnryb, "Orbits of Hurwitz action for coverings of a sphere with two special fibers", Indag. Math. (N.S.), 7:4 (1996), 549-558.

10. W. Fulton, "Hurwitz schemes and irreducibility of moduli of algebraic curves", Ann. of Math. (2), 10 (1969), 542-575.

11. T. Graber, J. Harris, J. Starr, A note on Hurwitz schemes of covers of a positive genus curve, arXiv: math/0205056.

12. J. S. Birman, "On braid groups", Comm. Pure Appl. Math., 22:1 (1969), 41-72.

13. E. Fadell, L. Neuwirth, "Configuration spaces", Math. Scand., 10 (1962), 111-118.

14. G.P. Scott, "Braid groups and the group of homeomorphisms of a surface", Proc. Cambridge Philos. Soc., 68:3 (1970), 605-617.

15. T. Graber, J. Harris, J. Starr, "Families of rationally connected varieties", J. Amer. Math. Soc., 16:1 (2003), 57-67.

Ф. Betpo (F. Vetro)

Поступило в редакцию

E-mail: vediba@libero.it 08.08.2011

Перевод с английского А.В. Домрина 